\title{
1-D KOCH FRACTAL ELECTROMAGNETIC BANDGAP MICROSTRIP STRUCTURES WITH R/A RATIOS HIGHER THAN 0.5
}

\author{
Juan de Dios Ruiz, Félix L. Martínez and Juan Hinojosa
}

\author{
Departamento de Electrónica, Tecnología de Computadoras y Proyectos \\ Universidad Politécnica de Cartagena \\ Plaza del Hospital $n^{\circ} 1$ \\ 30202 Cartagena (Murcia) -SPAIN
}

Corresponding Author: Dr. Juan Hinojosa, Email: juan.hinojosa@upct.es, Fax: +34 968326400

\begin{abstract}
A 1-D Koch fractal electromagnetic bandgap (KFEBG) microstrip structure is proposed. It is conceived by replacing the conventional holes etched in the ground plane of a microstrip line by level-1 Koch fractal cell geometries, which have been obtained from a hexagonal shape. In the case of the conventional 1-D EBG microstrip structure with periodic hole pattern, the design is limited to $r /$ a ratios lower than 0.45 , while the proposed pattern allows achieving $r / a$ ratios higher than 0.5. It is shown the conventional EBG and KFEBG microstrip structures behave as a stopband filter as $r / a \leq 0.45$. However, for $r / a=0.55$, the measurements have confirmed that the 1-D KFEBG microstrip structure presents a ultrawide stopband and, therefore, the proposed structure with $r / a>0.5$ can be useful for the design of low-pass filters.
\end{abstract}

Key words: Electromagnetic bandgap structures, filter; fractal structures; microstrip 


\section{INTRODUCTION}

The concept of photonic bandgap (PBG) structures was first introduced in Optics [1]. Later, it was scaled to microwave and millimeter-wave frequencies and the structures were designated as electromagnetic bandgap (EBG) [2]. The EBG structures are periodic structures, which exhibit a band of frequencies in which the electromagnetic propagation is not allowed. These structures can be used in numerous applications [3].

In microstrip technology, two-dimensional (2-D) structures with a periodic pattern etched in the ground plane were first proposed $[4,5]$. The periodic pattern was composed by holes, which created a stopband of the microwave signal transmission at the resonance frequency of the structure. The 2-D structure was then reduced to one-dimensional (1-D) structure, because the field levels are confined around the strip conductor and negligible outside of it [6-8]. In addition of the structure reduction to one-dimension, different periodic patterns with square, circular, triangular and sinusoidal shapes were used. All the designs with these different periodic patterns are limited to $r / a$ ratios lower than 0.5 and the frequency responses for $r / a$ ratios higher than 0.5 are unknown.

To solve this limitation, it is necessary to use a geometrical shape different from the above periodic patterns. Recently, fractal techniques have been used for the design of antennas and filters [9-15]. These fractal structures have a self-similar shape, which can be useful for the development of new patterns. Among these fractal techniques, no previous work has presented a frequency response with a $r / a$ ratio higher than 0.5 .

The aim of this work consists in the design of a 1-D EBG microstrip structure, which allows a periodic pattern in the ground plane with $r / a$ ratios higher than 0.5 . This kind of 1 D EBG microstrip structure is achieved by considering a periodic pattern based on level-1 Koch fractal element geometries, which have been obtained from a hexagonal shape. Two Koch fractal cell geometries were generated by using a method similar to the method of 
construction of fractal curves. By combining the two Koch fractal cells, several 1-D Koch fractal EBG microstrip structures with different $r / a$ ratio values were made. The simulated and measured results are compared with conventional EBG microstrip structures for $r / a$ ratios lower than 0.5 . Then, the simulation and experimental results of the 1-D Koch fractal EBG microstrip structure with $r / a=0.55$ are discussed.

\section{KOCH FRACTAL EBG MICROSTRIP STRUCTURE}

The conventional 1-D EBG microstrip structure is realized by etching a conductor microstrip line having a width of $50 \Omega$ on the top plane and several holes on the ground plane, as shown in Figure 1. The radius $r$ of all the holes and the distance $a$ between the centre of the holes are constant. The conventional 1-D EBG microstrip structure exhibits a bandgap when the Bragg reflection condition is satisfied [5-8]. The center frequency $\left(f_{0}\right)$ of the stopband is obtained from the distance $a$ between the centre of the holes. At $f_{0}$, the guided wavelength $\left(\lambda_{g}\right)$ is twice the period $a$. The design of conventional 1-D EBG microstrip structures is limited to $r / a$ ratios lower than 0.45 [8].

In order to have $r / a$ ratios higher than 0.5 , we have developed a new pattern based on Koch fractal curves. Figure 2 shows the construction process of the Koch curves up to the second iteration. The first curve, called $I_{0}$, is a straight line. The next fractal iteration $I_{1}$ is obtained by applying a scale factor of $1 / 3$. Thus, the initial straight line is partitioned into three equal parts. The segment at the middle is replaced with two others of the same length. The next fractal iterations are obtained iteratively. In each case, the overall length of the curve is $d$, while the total length of the wire is $l=d \cdot(4 / 3)^{n}$, where $n$ is the $n$th fractal iteration [9, 13].

The first Koch fractal iteration with a scale factor of $1 / 3$ was applied to a hexagonal 
shape. Two possible level-1 Koch fractal cell geometries were obtained. The two cells are shown in Figure 3 (the circles in dashed lines are a fictitious representation). Both Koch fractal cells present the same radius $r$. Thus, by combining and by etching the Koch fractal cells on the ground plane instead of the holes as in Figure 4, it is possible to achieve 1-D Koch fractal electromagnetic bandgap (KFEBG) microstrip structures with $r / a$ ratios lower and higher than 0.5 . The top view of the KFEBG microstrip structure is the same than the Figure 1(a).

\section{ANALYSIS OF THE KFEBG MICROSTRIP STRUCTURE}

Electromagnetic (EM) simulations and measurements have been carried out in order to compare the performance of the 1-D KFEBG microstrip structure with the conventional 1-D EBG (periodic holes) microstrip structure for different $r / a$ ratios lower than 0.5 . In order to not overload the figures, only EM simulation results of the conventional 1-D EBG microstrip structure are presented, since the measurements of such structure with different $r / a$ ratios have already been measured in previous works $[6,7]$. Then, the simulated and measured results of the 1-D KFEBG microstrip structure with $r / a=0.55$ have been analyzed. The RO3010 material of Rogers with a dielectric constant $\varepsilon_{r}=10.2(\operatorname{tg} \delta=0.0023$ at $10 \mathrm{GHz})$, substrate thickness $h=0.635 \mathrm{~mm}$, and copper thickness $t=17.5 \mu \mathrm{m}$ has been used as substrate for all KFEBG structures. The size of the microstrip was $40 \mathrm{~mm}$ wide and $147 \mathrm{~mm}$ long. The different structures have been designed with the purpose to have an operation frequency of $4.2 \mathrm{GHz}$ with the periodic value $a=14.1 \mathrm{~mm}\left(\lambda_{g}=2 a\right.$, where $\lambda_{g}$ is the guided wavelength in the unperturbed microstrip line) [6-8]. The total number of etched cells (holes or Koch fractal elements) has been set to $N=9$ as in $[6,7]$. At the top plane, the width of the conductor line was $W=0.594 \mathrm{~mm}$, and it corresponds to a $50 \mathrm{U}$ conventional microstrip line. The prototypes have been fabricated by means of a numerical milling machine. EM 
simulations and measurements have been obtained by using a commercially finite element simulator (HFSS) and a vector network analyzer (Agilent E5071B, $300 \mathrm{kHz}-8.5 \mathrm{GHz}$ ), respectively.

The simulated and measured results of the conventional 1-D EBG and 1-D KFEBG microstrip structures are shown in Figure 5 for two $r / a$ ratios: $r / a=0.25$ and $r / a=0.45$. As can be seen in Figure 5(a), the simulated conventional 1-D EBG microstrip structure with $r / a=0.25$ exhibits the characteristic of multi-stopband. The first stopband is centered at 4.2 $\mathrm{GHz}$ with a bandwidth of $2.1 \mathrm{GHz}$ (below $20 \mathrm{~dB}$ ), and the center frequency of the second stopband is twice as much of the center frequency of the first stopband. As the radius of the hole is increased $(r / a=0.45)$, the stopband becomes larger (Figure 5(b)). The results obtained from the proposed 1-D KFEBG microstrip structure present a similar behavior with a smaller stopband. It seems that the tendency as increases the $r / a$ ratio is to suppress the passband between both stopbands and to achieve a wider stopband. The simulated and measured results of the 1-D KFEBG microstrip structures $(r / a=0.25$ and $r / a=0.45)$ are in good agreement, except for the attenuation depth of the stopband with $r / a=0.45$ (Figure 5(b)). The difference is due to the substrate and metallic losses (they were considered lossless during the simulations), the performance of the connectors, the repeatability errors due to the assemblage of the different 1-D KFEBG microstrip structures and the limitation of the $S$ parameter measurements with the vector network analyzer.

Figure 6 shows the simulated and measured insertion losses $\left|S_{21}\right|$ of the 1-D KFEBG microstrip structure for $r / a=0.55$. In this design, the passband involved between both stopbands in the results with the previous structures $(r / a=0.25$ and $r / a=0.45)$ is suppressed. Moreover, it achieves a wide stopband and a high attenuation, which can be useful for the design of low-pass filter. The simulated and measured results are similar, except 
for the attenuation depth of the stopband. The discrepancy is due to the same causes that for the measurements of the 1-D KFEBG microstrip structure with $r / a=0.45$.

\section{CONCLUSION}

In this paper, a periodic pattern based on Koch fractal has been applied to a 1-D electromagnetic bandgap (EBG) microstrip structure. This periodic Koch fractal pattern, etched in the ground plane of the microstrip line, allows $r$ / $a$ ratios higher than 0.45 , which is the upper limit for the conventional 1-D EBG microstrip structure with holes etched in the ground plane. It is shown that the 1-D Koch fractal EBG (KFEBG) microstrip structures with $r / a$ ratios lower than 0.45 present similar bandstop filter responses as the conventional 1-D

EBG microstrip structures. However, as the $r / a$ ratios are increased above 0.5 , the 1-D KFEBG microstrip structures achieve low-pass filter responses of wide stopband. 


\section{ACKNOWLEDGEMENTS}

This work was supported by Ministerio de Educación y Ciencia of Spain (TEC2007-67630C03-02/TCM).

\section{REFERENCES}

1 E. Yablonovitch, Photonic band-gap structures, Journal Opt Soc Amer B 10 (1993), 283295.

2 Y. Rahmat-Samii, and H. Mosallaei, Electromagnetic band-gap structures: Classification, characterization and applications, in Proceedings Int Conf Antennas Propag, vol. 2, Manchester, Apr. 2001, 560-564.

3 P. de Maagt, R. Gonzalo, and J. C. Vardaxoglou, Review of electromagnetic bandgap technology and applications, Radio Science Bulletin 309 (2004), 11-24.

4 Y. Qian, V. Radisic, and T. Itoh, Simulation and experiment of photonic band-gap structures for microstrip circuits, in Proceedings Asia Pacific Microwave Conference, Hong Kong, Dec. 1997, 585-588.

5 V. Radisic, Y. Qian, R. Coccioli, and T. Itoh, Novel 2-D photonic bandgap structure for microstrip lines, IEEE Microwave Guided Wave Lett 8 (1998), 69-71.

6 M. A. G. Laso, M. J. Erro, D. Benito, M. J. Garde, T. Lopetegi, F. Falcone, and M. Sorolla, Analysis and design of 1-D photonic bandgap microstrip structures using a fiber grating model, Microwave Opt Tech Lett 22 (1999), 223-226.

7 F. Falcone, T. Lopetegi, and M. Sorolla, 1-D and 2-D photonic bandgap structures, Microwave Opt Tech Lett 22 (1999), 411-412. 
8 T. Lopetegi, M. A. Laso, M. J. Erro, D. Benito, M. J. Garde, F. Falcone, and M. Sorolla, Novel photonic bandgap microstrip structures using network topology, Microwave Opt Tech Lett 25 (2000), 33-36.

9 C. Puente-Baliarda, J. romeu, and A. Cardama, The Koch monopole: A small fractal antenna, IEEE Trans Antennas Propag 48 (2000), 1773-1781.

10 Y. Q. Fu, N. C. Yuan, and G. H. Zhang, A novel fractal microstrip PBG structure, Microwave Opt Tech Lett 32 (2002), 136-138.

11 K. Siakavara, Novel microwave microstrip filters using photonic bandgap ground plane with fractal periodic pattern, Microwave Opt Tech Lett 43 (2004), 273-276.

12 W. L. Chen, G. M. Wang, Y. N. Qi, and J. G. Liang, A novel wide stopband PBG structure with fractal features and its application to the design of microstrip low-pass filter, in Proceedings Int Symp Antennas Propag EM theory, Guilin, China, Oct. 2006.

13 A. L. P. S. Campos, E. E. C. de Oliviera, and P. H. F. Silva, Miniaturization of frequency selective surfaces using fractal Koch curves, Microwave Opt Tech Lett 51 (2009), 19831986.

14 P. Kurgan, and M. Kitilinski, Novel microstrip low-pass filters with fractal defected ground structures, Microwave Opt Tech Lett 51 (2009), 2473-2477.

15 M. Smierzchalski, P. Kurgan, and M. Kitlinski, Improved selectivity compact band-stop filter with gosper fractal-shaped defected ground structures, Microwave Opt Tech Lett 52 (2010), 227-229. 
Figure captions:

Figure 1 Conventional 1-D EBG microstrip structure with six holes etched in the ground plane. (a) Top view and (b) Bottom view.

Figure 2 Koch fractal curves: iterations $I_{0}$ through $I_{2}$.

Figure 3 Two level-1 Koch fractal cell geometries $\left(E_{1}\right.$ and $\left.E_{2}\right)$

Figure 4 Bottom view of the 1-D KFEBG microstrip structure with nine Koch fractal cells etched in the ground plane.

Figure 5 Simulated and measured $\left|S_{21}\right|$ parameters of the conventional 1-D EBG (periodic holes) and 1-D KFEBG microstrip structures. (a) $r / a=0.25$ and (b) $r / a=0.45$.

Figure 6 Simulated and measured $\left|S_{21}\right|$ parameters for the 1-D KFEBG microstrip structure with $r / a=0.55$. 
Figure 1

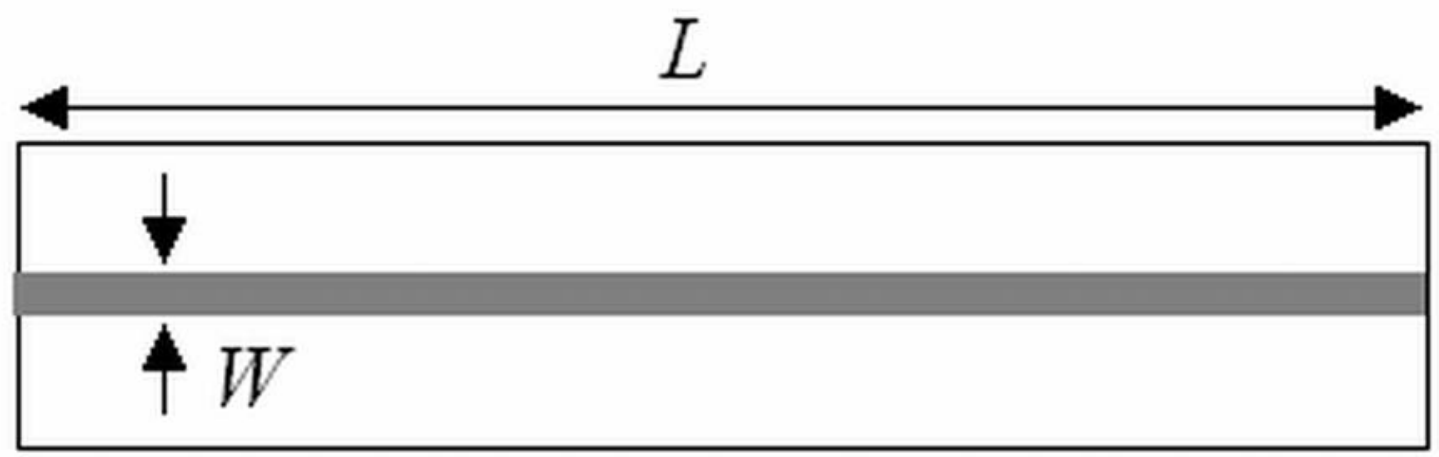

(a) 
Figure 1

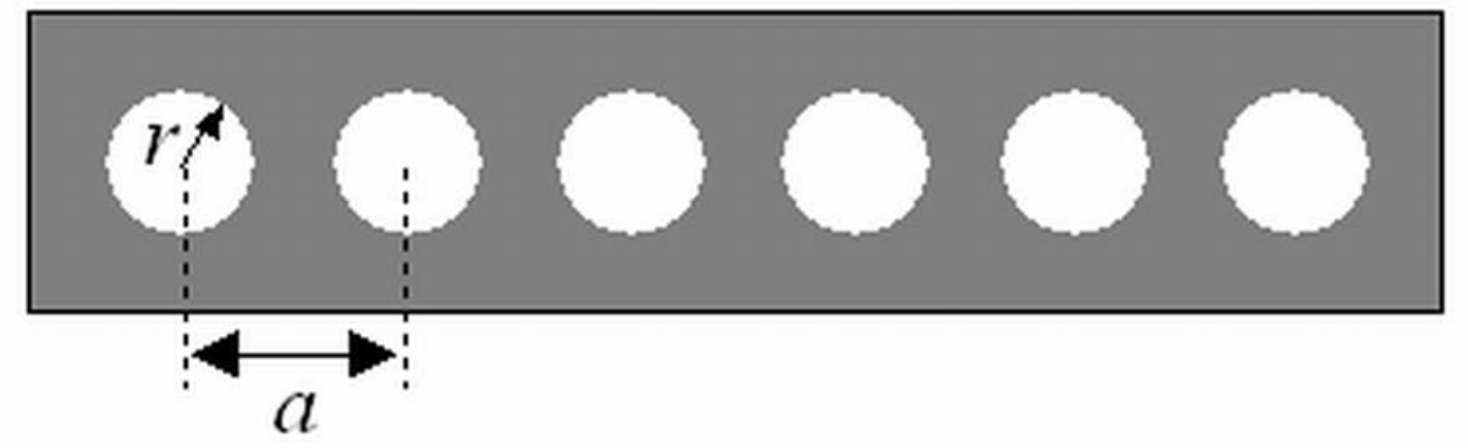

(b) 
Figure 2
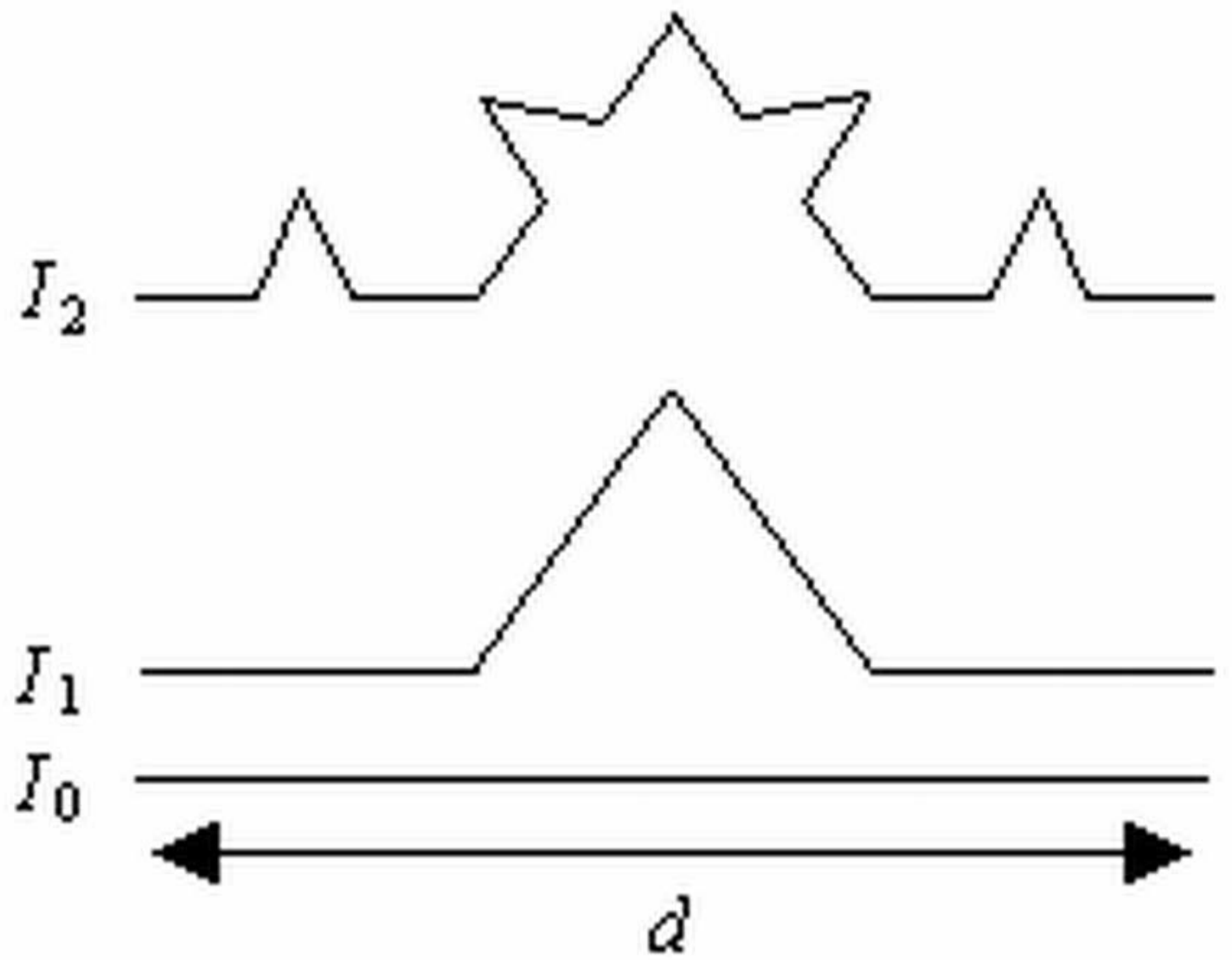
Figure 3

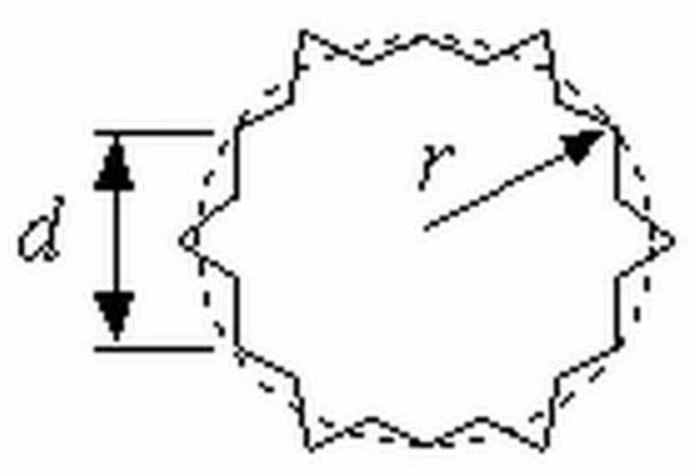

$E_{1}$

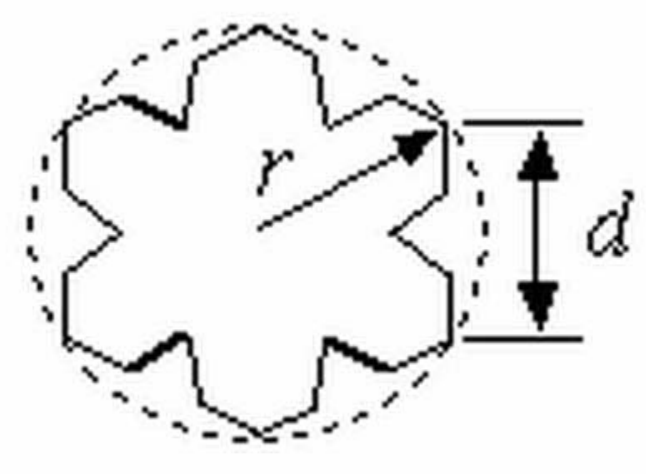

$E_{2}$ 
Figure 4

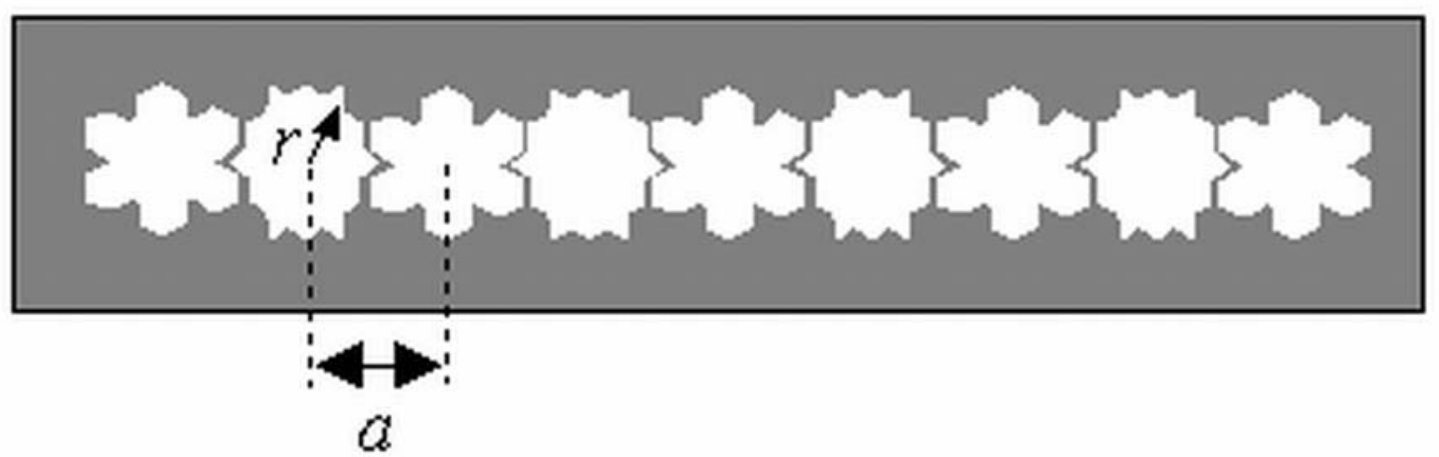


Figure 5

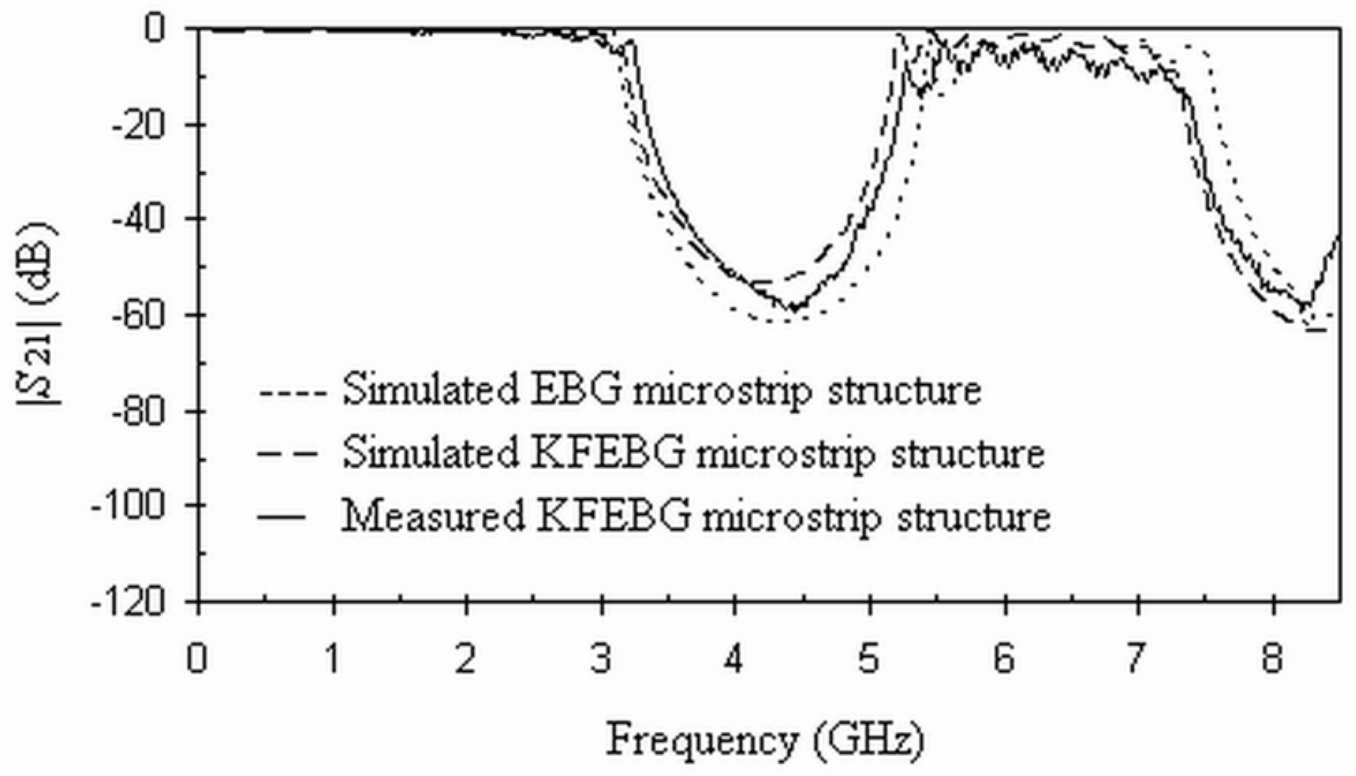

(a) 
Figure 5

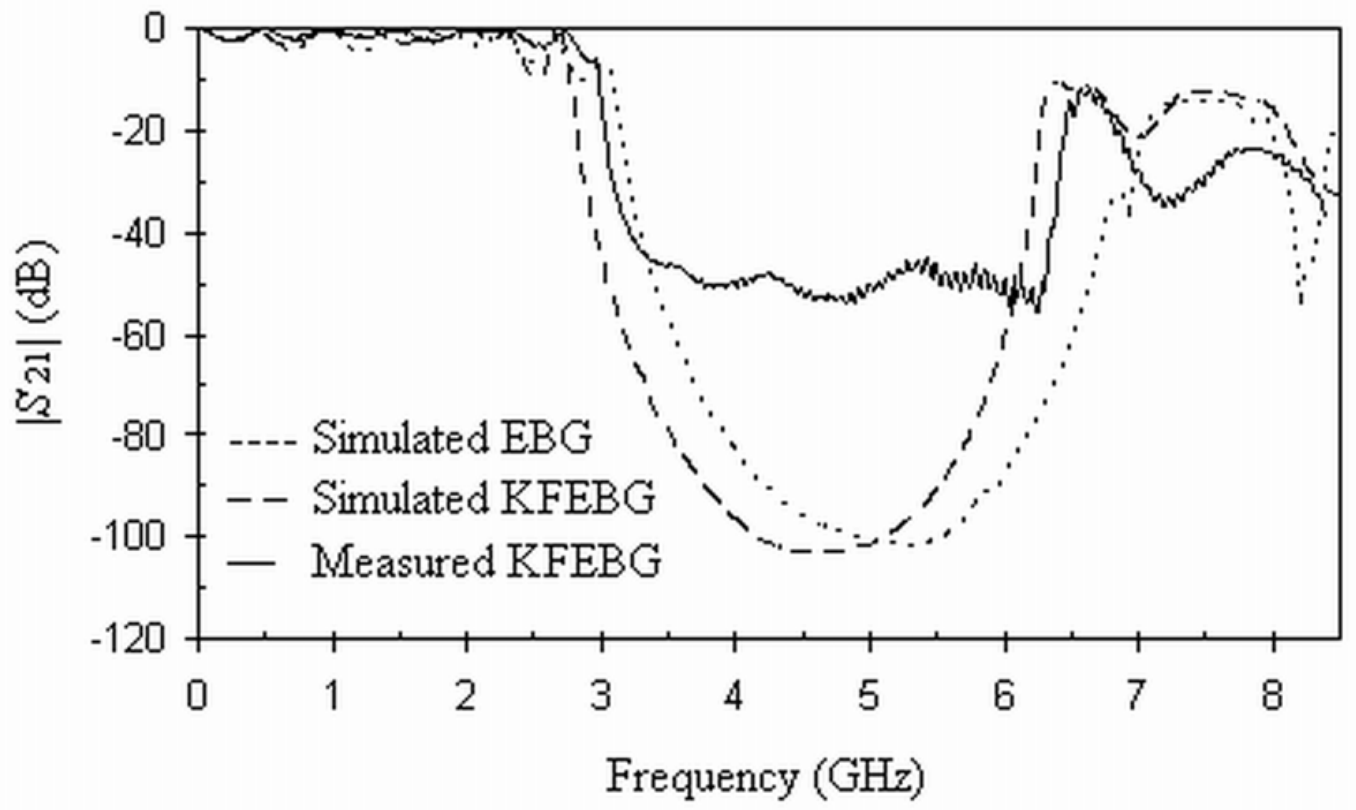

(b) 
Figure 6

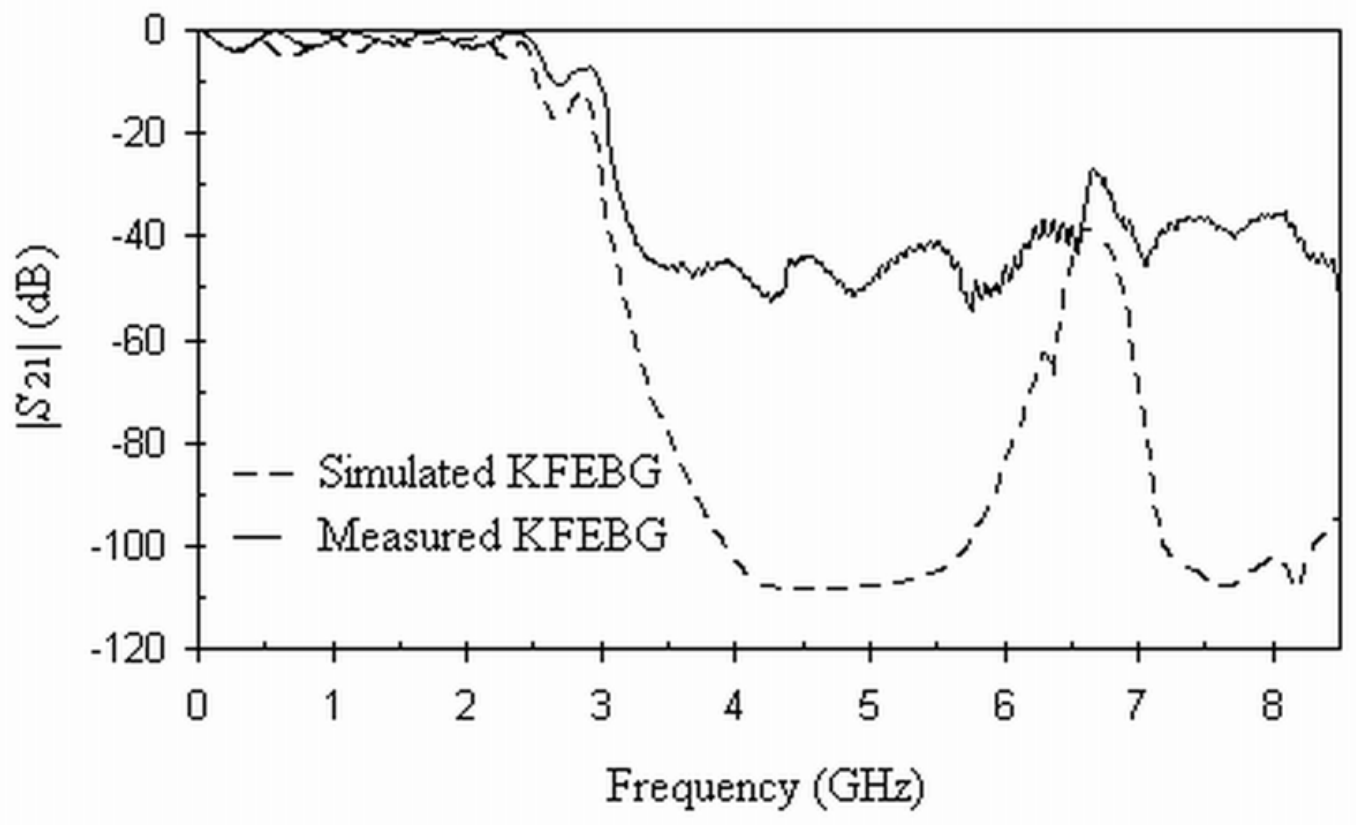

